\title{
Hypertension Induced by Hypothalamic Transplantation from Genetically Hypertensive to Normotensive Rats
}

\author{
R. Eilam, ${ }^{1}$ R. Malach, ${ }^{1}$ F. Bergmann, ${ }^{2}$ and M. Segal ${ }^{1}$ \\ 'Department of Neurobiology, The Weizmann Institute of Science, Rehovot 76100, Israel, and 'Department of \\ Pharmacology, The Hebrow University, Hadassah Medical School, Jerusalem 91010, Israel
}

\begin{abstract}
The role of the hypothalamus (HTH) in the pathogenesis of genetic hypertension was studied in spontaneously hypertensive rats (SHR). It is currently believed that, in this strain, the genetic defect manifests itself mainly in the HTH. We examined this hypothesis by grafting HTH neurons from embryos of SHR or control Wistar Kyoto (WKY) rats into the HTH of adult normotensive WKY rats. Changes in host systolic blood pressure (SBP) were monitored, and alterations in vasoactive intestinal polypeptide (VIP) gene expression of the host brain were studied.

In rats grafted with HTH tissue from SHR embryos (G-SHR), the blood pressure rose by $31 \%$ as compared with that in the grafted control group. The blood pressure climbed gradually over a period of 6 weeks to its highest level, which was maintained for at least 3 months following gratting. Along with the elevated blood pressure, the heart weight increased by $80 \%$ compared to controls. Behavioral changes were also evident in the G-SHR rats, and these were similar to those of the native SHR strain. In situ hybridization histochemistry showed a $40 \%$ elevation in VIP transcripts in the suprachiasmatic nucleus of the host G-SHR brain compared to controls. These studies demonstrate that transplantation of embryonic SHR HTH tissue into brains of adult normotensive rats results in the development of hypertensive characteristics in the host. It thus appears that the HTH is a prime candidate for the source of changes leading to spontaneous hypertension in mammals.
\end{abstract}

Genetic selection of the spontaneously hypertensive rat strain (SHR), in which there is a gradual development of hypertension as a function of age, represents a breakthrough in blood pressure (BP) research. This strain serves as a model for the study of the effects of hypertension on various systems. It is currently believed that the genetic defect in SHR manifests itself mainly in the hypothalamus (HTH; Fukushima, 1968; Nelson and Boulant, 1981).

\footnotetext{
Received Jan. 30, 1990; revised Sept. 10, 1990; accepted Sept. 13, 1990.

We wish to thank Dr. R. Avidor and Professor I. Gozes for their help with the in situ work and Professor A. Biegon for the use of her computerized image analysis system. This work was supported by the Levi Eshkol Foundation, The National Council for Research and Development Israel, the Israel Institute for Psychobiology, Charles E. Smith Family Foundation, and the Israeli Ministry of Health Foundation.

Correspondence should be addressed to Dr. R. Eilam, Department of Neurobiology, Perlman Building, The Weizmann Institute of Science, Rehovot 76100 , Israel.

Copyright (C) 1991 Society for Neuroscience $0270-6474 / 91 / 110401-11 \$ 03.00 / 0$
}

The hypertensive rats are characterized by a broad spectrum of neuroendocrine deviations from normal rats. In our previous work, we found an increase in vasoactive intestinal polypeptide (VIP) mRNA transcripts in the suprachiasmatic nucleus (SCN) of SHR (Avidor et al., 1989). It has also been demonstrated that, in the SHR strain, the hypothalamic and brain-stem concentration of vasopressin (VP) is reduced (Lang et al., 1981; Morris and Keller, 1982). However, the basal levels of adrenergic amines such as epinephrine, angiotensin II (Nagaoka and Lovenberg, 1977; Morris et al., 1981), and digitalislike substances (Millett et al., 1986) are higher in the HTH of the hypertensive rat than in that of the normotensive animal. Metabolic research demonstrated a higher level of activity in the HTH of SHR during the development of hypertension, compared with those observed in the Wistar Kyoto strain (WKY; Krukoff and Weigel, 1989). Histological studies have revealed that hypertension is associated with changes in the hypothalamoneurohypophyseal system, with a significant reduction in the size of the paraventricular nucleus (PVN; Nelson and Boulant, 1981). Induction of hypertension in rats by different methods is associated with reduced responses to noxious thermal stimuli or to noxious mechanical stimuli (Zamir et al., 1980). The underlying mechanisms responsible for this effect have not been fully clarified. However, it is unlikely that the diminished responsiveness to noxious stimuli results from a pathological change in primary nociceptor afferent activity, secondary to hypertension, because the hypoalgesic response is observed following acute elevation in BP, and is also present at the onset of the development of hypertension in SHR (Faden and Feuerstein, 1983; Randish and Maixner, 1984).

The objectives of the present study are to examine the relationship between the HTH and hypertension in the SHR strain through the use of brain grafts. HTH tissue from embryos of SHR or control WKY rats was grafted into the HTH of adult normotensive WKY rats. Subsequent changes in host systolic blood pressure (SBP) were followed for 3-12 months thereafter. In addition, behavioral and physiological changes that characterize SHR were examined in the grafted rats. These changes include myocardial hypertrophy, hypoalgesia, and alterations in VIP gene expression in the host brain. Graft development was studied by immunohistochemical staining for VIP and VP.

\section{Materials and Methods}

Implantation. Pregnant dams were killed by cervical dislocation. Their 16-d-old embryos were dissected out under sterile conditions and placed in an oxygen-enriched, chilled L15 solution, as described elsewhere (Segal et al., 1989). Using a stereomicroscope and fine dissecting tools, the ventral boundaries of the HTH were clearly visible. The HTH was separated from the septal area by a coronal cut at the level of the chiasma, 


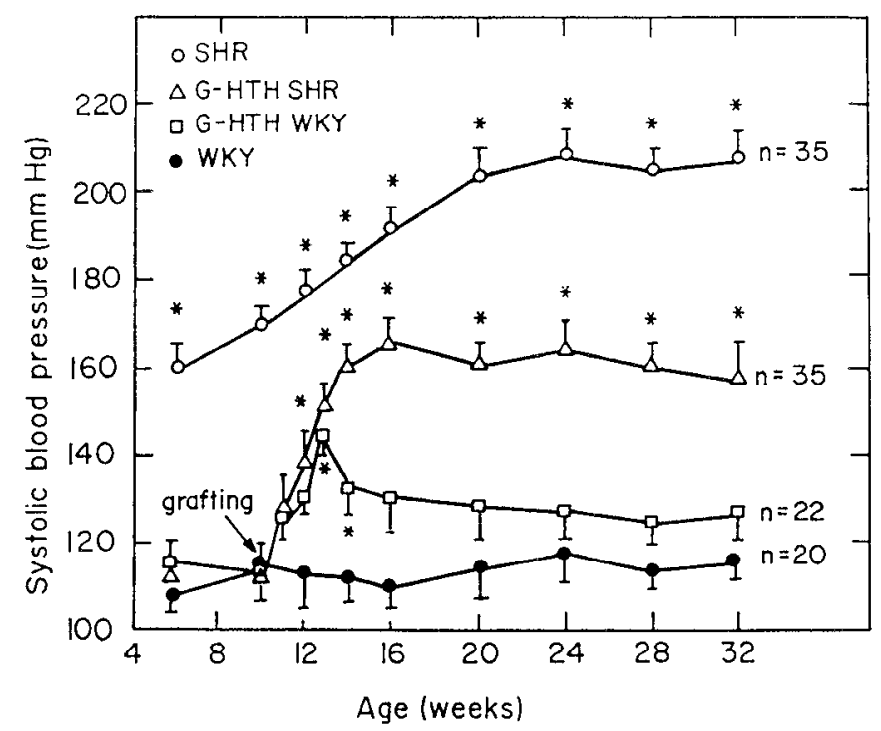

Figure 1. Changes in SBP ( \pm SEM) as function of age. Significant differences between experimental groups and controls are shown by asterisks (ANOVA; ${ }^{*}, p<0.05$ ).

from the midbrain by a coronal cut at the level of the mammillary bodies, and from the pyriform cortex and ventral forebrain by 2 sagittal sections. The HTH was separated from the thalamus by a horizontal cut about $1 \mathrm{~mm}$ from the ventral surface and stored in ice-cold L15 medium until use. The tissue was dissociated mechanically with a pair of forceps without the addition of fluid and suctioned into a $10-\mu \mathrm{l}$ Hamilton syringe. Individual HTH pieces, in a volume of $2-3 \mu \mathrm{l}$, were injected stereotaxically into the medial HTH region (third ventricle) of the host rats.

Adult (250-300 gm) WKY host rats were anesthetized (3.5\% chloral hydrate, $1 \mathrm{cc} / 100 \mathrm{gm}$ ) and placed in a stereotaxic frame. HTH tissue from SHR embryos was transplanted into 35 adult normotensive (WKY) rats (G-SHR). In a similar procedure, HTH taken from WKY embryos was implanted into 22 WKY rats (G-WKY). Sham operations were performed on $20 \mathrm{WKY}$ normotensive rats. In additional control experiments, 5 WKY rats were grafted with hippocampal tissue taken from 16-d-old SHR embryos (G-HPC SHR).

Systolic blood pressure. Systolic blood pressure (SBP) was measured by tail plethysmography in conscious rats that were subjected to the procedure twice prior to experimentation (Crofton et al., 1978). The rats were warmed to $37^{\circ} \mathrm{C}$ for $20 \mathrm{~min}$ and then placed in an animal warming unit to maintain tail arterial vasodilation. Recordings were taken with a pneumatic pulse transducer, connected to a physiograph. In measuring intra- and intergroup SBP, the rats were chosen at random. The mean pressure is presented as the average of 4 measurements for each rat.

Assessment of pain sensitivity. A copper plate, surrounded by a Plexiglas cage and maintaincd at $53 \pm 1^{\circ} \mathrm{C}$ with circulating warm water, was covered with an isolating cardboard. Each rat was placed on the cardboard for $1 \mathrm{~min}$ before the cardboard was removed. The latency to licking one of the hind paws in reaction to the evoked pain was recorded. Each rat was removed from the cage immediately after the paw lick. If no licking took place for $45 \mathrm{sec}$, the animal was removed from the cage, and the result was scored as 45 sec.

Body and heart weight. Upon completion of the experiments, the animals were weighed, anesthesized, and perfused with $4 \%$ paraformaldehyde in phosphate-buffered saline. The hearts were then excised, washed with saline, blotted, and weighed.

Immunohistochemistry. Ten G-SHR, 4 G-WKY, and 10 sham-operated rats, all chosen at random from the physiologically tested groups, were used in the immunohistochemical studies. The animals were killed 1-12 months following grafting, to check the viability of the transplanted tissue as well as other effects of the surgical procedure. In 9 of 10 G-SHR rats, SBP was found to be higher than that recorded in the control group.

Following perfusion of the anesthesized animals, the brains were removed and stored for $5-14 \mathrm{~d}$ in $1 \%$ paraformaldehyde. Serial $100-\mu \mathrm{m}$ coronal sections of the implanted region were placed in wells containing
$0.05 \%$ Tris buffer $(\mathrm{pH}, 7.4)$. They were then washed 4 times $(5 \mathrm{~min}$ each) in Tris buffer to remove excess paraformaldehyde. Selected sections were stained for Nissl substance while the rest were processed for immunocytochemistry using the following steps: The sections were incubated for $15 \mathrm{~min}$ in $0.3 \%$ hydrogen peroxide to reduce endogenous peroxidase activity. After an additional wash, the sections were incubated for $2 \mathrm{hr}$ in blocking medium containing 20\% normal goat serum (Vector Laboratories, Burlingame, CA), and then were incubated overnight at room temperature in primary antisera to either VIP $(1: 1000)$ or VP $(1: 3000 ;$ Incstar Corp., Stillwater, MN) with constant agitation. The reaction was carried out using the avidin-biotin peroxidase technique (ABC Vectastain, Vector Laboratories, Burlingame, CA). Preincubation overnight with synthetic peptides, VIP $(165 \mu \mathrm{g} / \mathrm{ml})$ or VP $(100$ $\mu \mathrm{g} / \mathrm{ml}$ ), of selected sections from different levels in the hypothalamus (i.e., anterior commissural nucleus, anterior PVN, and medial PVN) completely eliminated staining in control sections. In addition, sections from the same levels were preincubated with synthetic oxytocin (200 $\mu \mathrm{g} / \mathrm{ml}$ ) to examine whether positive staining resulted from VP-oxytocin cross-reactivity (see Results).

In situ hybridization. Five months after grafting, experimental and control animals were killed, and their brains were removed and frozen. Twenty-micron-thick frozen cryostat sections were mounted on glass slides. The sections were then submitted to an in situ hybridization procedure using a technique described previously (Bloch et al., 1986), with slight modifications as previously described (Gozes et al., 1989). A 30-base synthetic, single-strand oligonucleotide DNA probe was used. The specificity of hybridization of the probe has been established previously (Eckenstein and Baughman, 1984). Probes were labeled with $\alpha$-P ${ }^{32}$-deoxycytidine triphosphate (New England Nuclear; $3000 \mathrm{Ci} / \mathrm{mmol}$ ) by terminal deoxynucleotidyl transferase. Using this technique, we could detect VIP mRNA only in the SCN, and not in other parts of HTH (Avidor et al., 1989; Gozes et al., 1989).

Autoradiograms were analyzed with the aid of an IBM-PC-based computerized image analysis system. The system includes PC-vision, a digitized board (Imaging Technology Inc.), and customized software (Biegon and Israeli, 1978). Standardization curves derived from microscale standards were used to convert gray-level reading of the autoradiograms into optical density. The average values of the areas showing positive hybridization were calculated. Specific background reading was subtracted from the total reading in each section measured.

Statistical analyses. Results of SBP and pain sensitivity measurements of control and G-SHR groups were compared by analysis of variance (ANOVA). The other results were compared using the paired and unpaired Student's $t$ test when appropriate. Values are presented as mean \pm SEM.

\section{Results}

\section{Blood pressure}

In the sham-operated WKY rats, SBP remained constant throughout the experiment (Fig. 1). The control group G-HPC SHR exhibited no significant changes in $\mathrm{BP}$, which was essentially similar to those of the sham-operated group (not shown). The SBP of the G-WKY group rose from the $112 \pm 5 \mathrm{~mm} \mathrm{Hg}$ preimplantation level to $131 \pm 4 \mathrm{~mm} \mathrm{Hg} 2$ weeks after implantation. A maximal value $(F=12.59 ; p<0.05)$ of $144 \pm 5$ $\mathrm{mm} \mathrm{Hg}$ was recorded 3 weeks after grafting and gradually dropped within the following 4 weeks to the level of the sham-operated WKY rats.

In the first few weeks following implantation, the SBP of the G-SHR rats climbed rapidly from the control WKY level (115 $\pm 4 \mathrm{~mm} \mathrm{Hg}$ ), reaching $165 \pm 7 \mathrm{~mm} \mathrm{Hg}$ after 6 weeks. This value then remained constant during the remainder of the experiment. After the fourth week after implantation, the SBP was significantly higher in this group than in the G-WKY rats, though lower than the respective values of the native SHR group $(F=$ $19.41 ; p<0.05)$.

In the SHR groun, the SBP rose steadily from the $160 \pm 5$ $\mathrm{mm} \mathrm{Hg}$ value recorded at the age of 6 weeks, reaching $203 \pm$ $7 \mathrm{~mm} \mathrm{Hg}$ at 20 weeks of age. This value remained constant for 


\section{Time after grafting (weeks)}
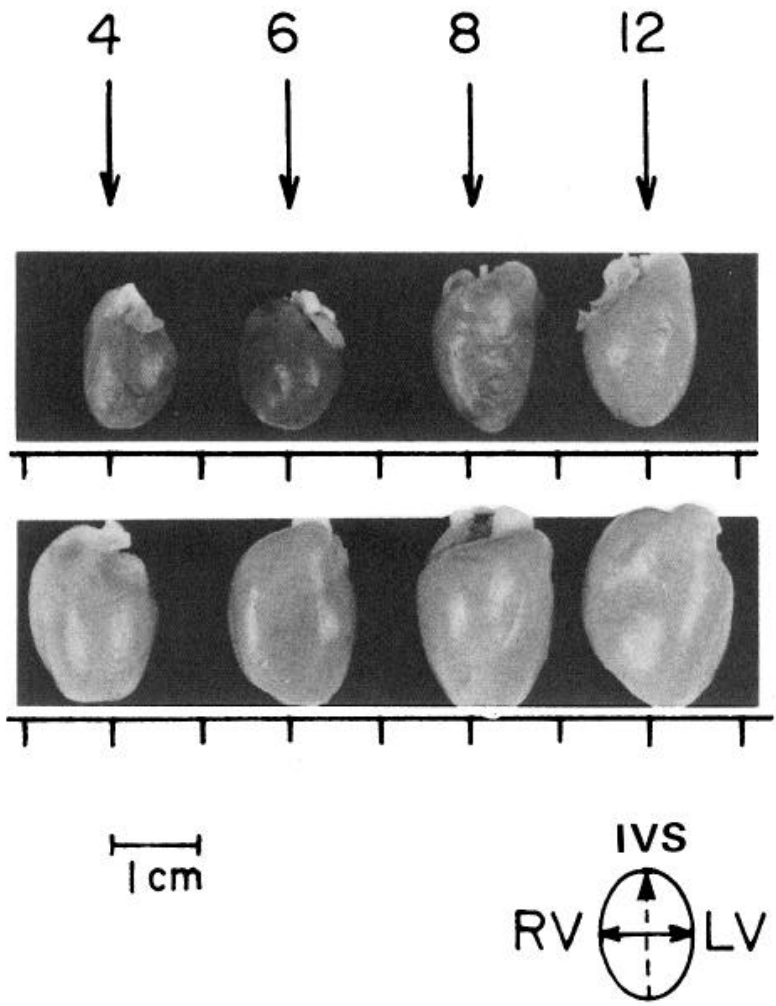
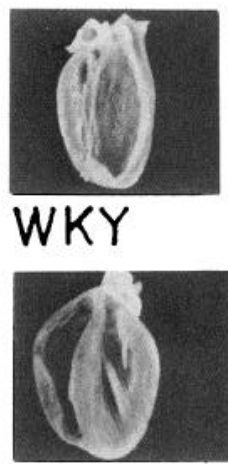

G-SHR
Figure 2. Increase in heart volume with time after grafting. Upper panel, Hearts of sham-operated WKY rats. Lower panel, Hearts of rats grafted with HTH tissue from SHR embryos. The elevation in heart weight of G-SHR was followed by marked increase in the width of the left ventricle wall and the interventricular septum (right panel). $R V$, right ventricle; $L V$, left ventricle; $I V S$, interventricular septum. the duration of the experiment. Throughout the experiment, the SBP of this group was markedly higher $(F=22.43 ; p<0.05)$ than that of any of the other groups.

\section{Heart and body weight}

Between 4 and 6 weeks after transplantation, relative and absolute heart weight were higher in the G-WKY group $(n=4)$ than in the age-matched sham-operated WKY rats $(n=6$; Table 1). In the G-SHR group, heart weight and volume remained higher than that of the WKY and SHR rats throughout the entire 3-month experimental period. The increase in heart weight was caused by a marked increase in the width of the left ventricle walls and the interventricular septum (Fig. 2).

\section{Pain sensitivity}

The latency of licking one of the hind paws was essentially the same in the control groups G-WKY and WKY (Fig. 3). In contrast, the paw-lick latency was significantly longer in the G-SHR group, increasing from $14 \pm 2 \mathrm{sec}$ prior to implantation to 28 $\pm 3 \sec (F=20.46 ; p<0.05) 4$ weeks after grafting, when it dropped slightly.

\section{In situ hybridization}

Figure 4 shows serial-section autoradiograms in which hybridization to the SCN of brain sections of the control G-SHR and SHR groups is compared, as measured by optical density. The absorbance values of VIP mRNA $\times 100$, in increasing order of magnitude, were as follows: WKY = G-HPC SHR $<$ G-WKY $<$ G-SHR < SHR (Table 2). The increase in VIP mRNA corresponded to the elevation of $\mathrm{BP}$ observed.

\section{Immunohistochemistry}

Nine of the 10 examined grafts survived. The viable grafts were located either within (7) or adjacent to (2) the third ventricle. The nonviable graft, found in the G-SHR rat that showed no symptoms of hypertension, was located within the $\mathrm{HTH}$, albeit at a distance from the third ventricle, near the lateral HTH nucleus. Similar increases in SBP $(164 \pm 7$ and $169 \pm 8 \mathrm{~mm}$ $\mathrm{Hg}$ ) were recorded in the animals that had received the viable grafts at different locations in the HTH.

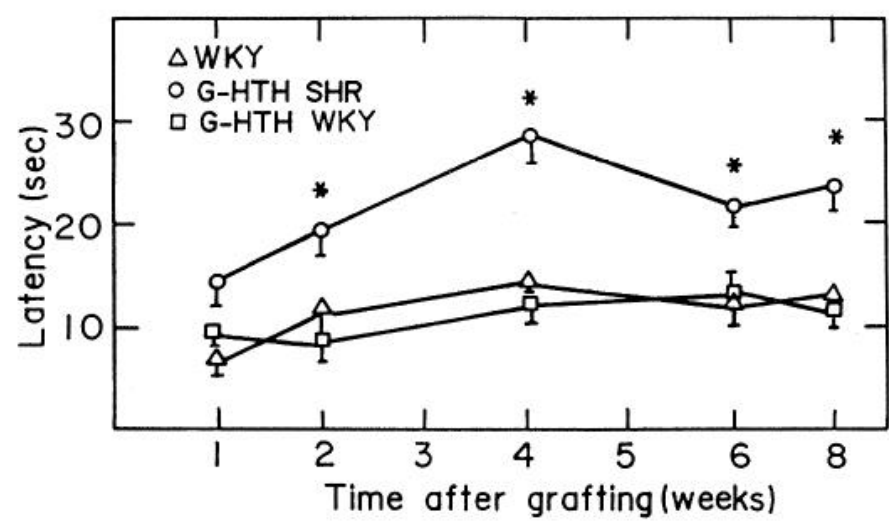

Figure 3. Latency $( \pm \mathrm{SEM})$ of rats licking one hind paw in response to pain, evoked by hot plate, as function of time after grafting. Significant differences between groups are shown by asterisks ( $n=6$ for all groups; ANOVA; $\left.{ }^{*}, p<0.05\right)$. 


\begin{tabular}{|c|c|c|c|c|}
\hline $\begin{array}{l}\text { Time after } \\
\text { grafting }\end{array}$ & WKY/cont & G-WKY & G-SHR & SHR \\
\hline \multicolumn{5}{|l|}{ Four weeks } \\
\hline BW & $260 \pm 7.2$ & $270 \pm 11.4$ & $275 \pm 9.3$ & $285 \pm 7.3$ \\
\hline HW & $0.95 \pm 0.12$ & $1.15 \pm 0.09^{*}$ & $1.24 \pm 0.14^{* *}$ & $1.17 \pm 0.1$ \\
\hline$n$ & 6 & 4 & 5 & 5 \\
\hline \multicolumn{5}{|l|}{ Six weeks } \\
\hline BW & $280 \pm 8.3$ & $283 \pm 7.2$ & $295 \pm 4.3$ & $295 \pm 4.2$ \\
\hline HW & $1.00 \pm 0.11$ & $1.22 \pm 0.14^{*}$ & $1.46 \pm 0.13^{* *}$ & $1.23 \pm 0.3^{*}$ \\
\hline$n$ & 6 & 4 & 4 & 5 \\
\hline \multicolumn{5}{|l|}{ Eight weeks } \\
\hline BW & $296 \pm 8.4$ & $310 \pm 15.2$ & $320 \pm 7.2$ & $328 \pm 4.3$ \\
\hline HW & $1.15 \pm 0.10$ & $1.10 \pm 0.09$ & $1.77 \pm 0.19^{* *}$ & $1.43 \pm 0.2^{* *}$ \\
\hline$n$ & 6 & 3 & 5 & 5 \\
\hline \multicolumn{5}{|c|}{ Twelve weeks } \\
\hline BW & $310 \pm 5.5$ & $320 \pm 10.5$ & $332 \pm 7.5$ & $340 \pm 12.8$ \\
\hline HW & $1.21 \pm 0.13$ & $1.13 \pm 0.10$ & $2.12 \pm 0.23^{* *}$ & $1.65 \pm 0.2^{* *}$ \\
\hline$n$ & 6 & 2 & 2 & 5 \\
\hline
\end{tabular}

This table shows body weight (BW, gm) and heart weight $(\mathrm{HW}, \mathrm{gm})$ in control Wistar Kyoto (WKY/cont) rats, normotensive rats grafted with HTH tissue from WKY embryos (G-WKY), normotensive rats grafted with HTH tissue from SHR embryos (G-SHR), and spontaneously hypertensive rats (SHR). $n$, number of animals used. Data are means \pm SEM. *, $p<0.05 ; * *, p<0.01$.

Neurons and fibers staining for VIP and VP were present in the surviving G-SHR grafts (Figs. 5, 6). In contrast, in the G-WKY grafts, only parvocellular neurons were noticed (Fig. $6 B$ ). Some of the fibers extending from the VIP- and VP-positive neurons appeared to penetrate the host brain (Fig. 6C,D), indicating the integration of the grafts in the host tissue. These immunochemical results were also supported by the appearance of Nissl-stained neuronal cell bodies in the transplant (Fig. 8). Furthermore, VP antibody in the presence of synthetic oxytocin, in a concentration that, according to manufacturer's specifications, should be sufficient to block activity, revealed both neuronal bodies and fibers (Fig. 7) in the HTH nuclei examined. However, we cannot rule out the possibility that the antiserum will stain for oxytocin elsewhere in the brain.

Upon examination of coronal sections, there was a considerable variation in graft size $(430-870 \mu \mathrm{m})$. The anterior-posterior extent of the graft ranged between 600 to $900 \mu \mathrm{m}$.

\section{Discussion}

To date, brain transplantation experiments have focused on the restoration of impaired host function by grafting normal tissue to replace nonfunctioning or malfunctioning areas of the brain (Boer et al., 1985). In contrast, our approach was to transplant putatively abnormal brain tissue, in an attempt to identify the responsible brain region and study the mechanism(s) involved in the induction of hypertension. We found that $90 \%$ of examined grafts remained viable even $1 \mathrm{yr}$ following transplantation, as judged by the presence of neurons within the graft stained for VP and VIP.

The regulation of arterial $\mathrm{BP}$ is maintained through interactions among several organs. Until now, the only isolated factor known to cause hypertension was the kidney (Bianchi et al., 1974).

Because the CNS (Calaresu et al., 1975), mainly the HTH

Table 2. SBP and absorption values $(A)$ of VIP mRNA

\begin{tabular}{|c|c|c|c|c|c|c|}
\hline \multirow[b]{2}{*}{$\begin{array}{l}\text { Experiment } \\
\text { Number }\end{array}$} & \multicolumn{2}{|l|}{ WKY } & \multicolumn{2}{|c|}{ G-HPC SHR } & \multicolumn{2}{|l|}{ G-WKY } \\
\hline & SBP & $\begin{array}{l}A \text { for VIP } \\
\text { mRNA } \times 100\end{array}$ & SBP & $\begin{array}{l}A \text { for VIP } \\
\text { mRNA } \times 100\end{array}$ & SBP & $\begin{array}{l}A \text { for VIP } \\
\text { mRNA } \times 100\end{array}$ \\
\hline 1 & 115 & 36.61 & - & - & 125 & 40.33 \\
\hline 2 & 125 & 32.43 & - & - & 130 & 35.84 \\
\hline 3 & 115 & 34.28 & 120 & 32.20 & 140 & 35.08 \\
\hline 4 & 120 & 33.24 & 130 & 31.56 & 125 & 37.78 \\
\hline 5 & 110 & 34.87 & 125 & 34.25 & 120 & 36.23 \\
\hline Mean \pm SEM & $117 \pm 2.3$ & $34.28 \pm 0.7$ & $125 \pm 2.9$ & $32.69 \pm 0.8$ & $128 \pm 3.0$ & $37.05 \pm 0.9^{*}$ \\
\hline
\end{tabular}




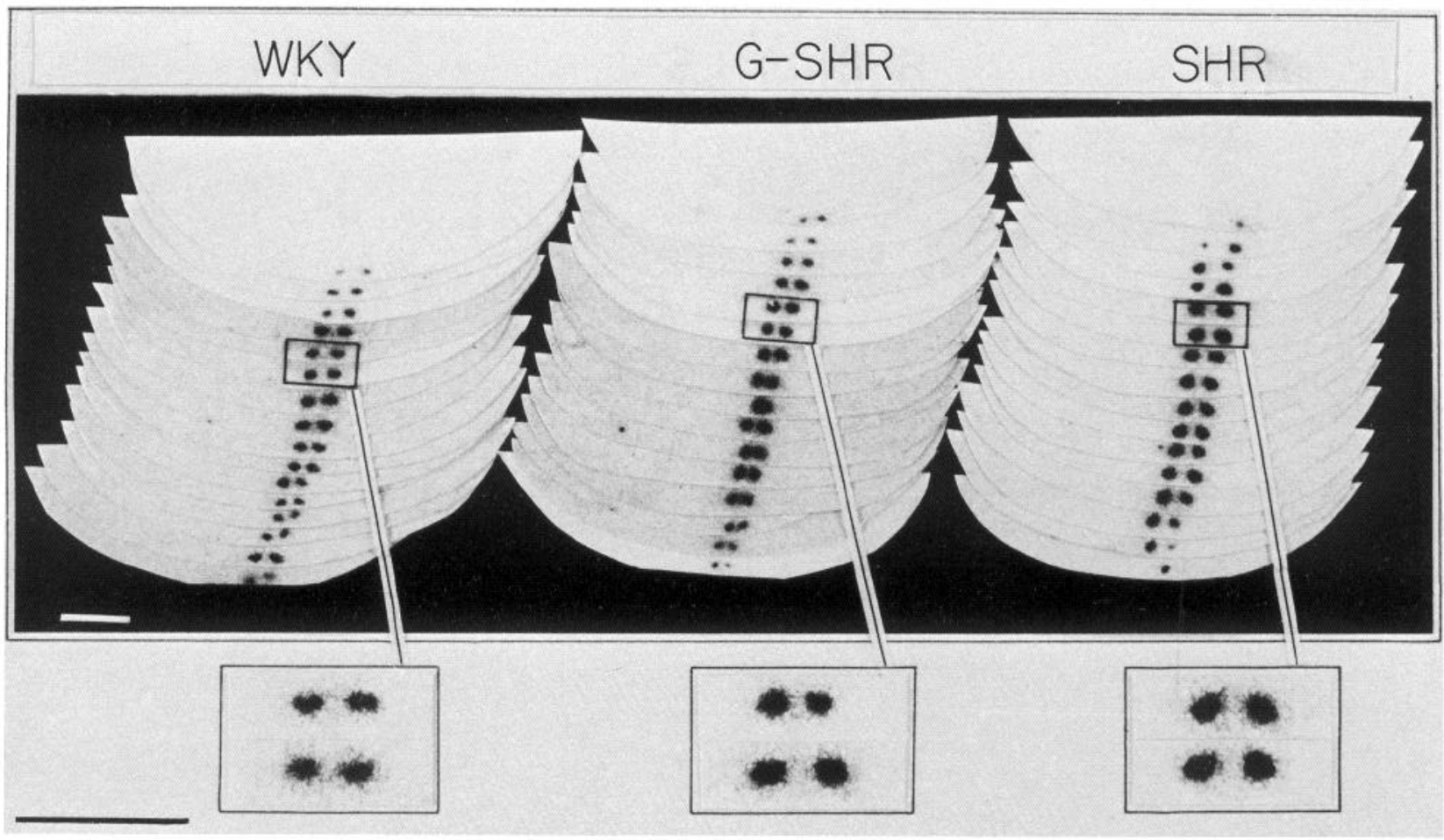

Figure 4. Labeling by VIP-specific probe in HTH SCN of 26 -week-old WKY rats, SHR, and G-SHR. Serial sections ( $20 \mu \mathrm{m}$ thick) of the HTH area beginning at the anterior part of the SCN are shown. The tissues in the square area are shown at a larger magnification at the bottom. Scale bars, $2 \mathrm{~mm}$.

(Swanson and Sawchenko, 1980), plays a dominant role in the central regulation of BP, a set of experiments was designed to determine whether it could, by itself, induce hypertension. Using the HTH transplantation technique, the results of our work showed that a grafted HTH from a hypertensive rat (SHR) could cause the development of the pathological condition in normotensive (WKY) rats. The predominant involvement of the HTH in producing hypertension in our model leads us to believe that this isolated organ is capable of inducing the disease and its major complication, cardiac hypertrophy.

Although our main finding was the induction and maintenance of hypertension in rats grafted with HTH tissue from SHR embryos (G-SHR), an additional phenomenon that bears men-

\begin{tabular}{lllll}
\hline $\begin{array}{l}\text { Table 2. } \\
\text { G-SHR }\end{array}$ & Extended & & \\
\cline { 1 - 2 } SBP & $\begin{array}{l}A \text { for VIP } \\
\text { mRNA } \times 100\end{array}$ & & SBP & $\begin{array}{l}A \text { for VIP } \\
\text { mRNA } \times 100\end{array}$ \\
\hline 170 & 46.52 & 205 & 50.38 \\
165 & 41.89 & 185 & 46.35 \\
180 & 44.56 & 210 & 47.98 \\
160 & 45.08 & 195 & 45.24 \\
165 & 42.89 & - & - \\
$168 \pm 3.0$ & $44.18 \pm 0.8^{*}$ & $198 \pm 4.8$ & $47.08 \pm 0.92^{*}$ \\
\hline
\end{tabular}

tioning was found, namely, the transient increase in BP induced in normotensive rats grafted with normal HTH tissue (G-WKY). This phenomenon was not observed in control WKY rats grafted with hippocampal tissue of hypertensive rats (G-HPC SHR). The results suggest that excess HTH tissue, by itself, may induce temporary hypertension. Because, in all 3 experimental groups (G-SHR, G-WKY, and G-HPC SHR), the grafted tissue remained viable after $1 \mathrm{yr}$, it seems that the difference between the G-SHR and G-WKY animals was the ability of the latter to restore BP to normal level after 6 weeks. In contrast, BP in the G-SHR rats remained elevated after this period, continuing to climb in the following 22 weeks.

The short duration of the hypertension induced by normal HTH tissue may be explained by a pressor effect of the excess tissue, later compensated for by other regulatory mechanisms. Long-term regulatory mechanisms that act to balance arterial BP are mostly related to kidney function (Guyton, 1987). It is possible that the long-lasting hypertension induced in the G-SHR rats is due to impairment of an HTH effect on distant peripheral BP-controlling organs, including the kidneys, through neural or endocrine pathways. Here, the compensatory mechanisms were seemingly ineffective. Further experiments are required to study this hypothesis.

However, it should be noted that, apart from the HTH, several brain-stem nuclei associated with cardiovascular regulation have been implicated in the development and maintenance of hypertension. Alteration in catecholamine content and turnover have been reported in regions of the HTH and brain-stem nuclei, particularly during the developmental phase of hypertension (Versteeg et al., 1976; Saavedra et al., 1978; Wijnen et al., 1978). 
Figure 5. Transplant of embryonic day 16 SHR donor $(T)$ placed between SCN and SON. Adjacent sections were stained for VP $(A)$ and VIP $(B)$. $m$, magnocellular neurons; $O X$, optic chiasm; $p$, parvocellular neurons. The graft contains parvocellular VP-positive neurons and resembles the SCN. Scale bars, $500 \mu \mathrm{m}$.
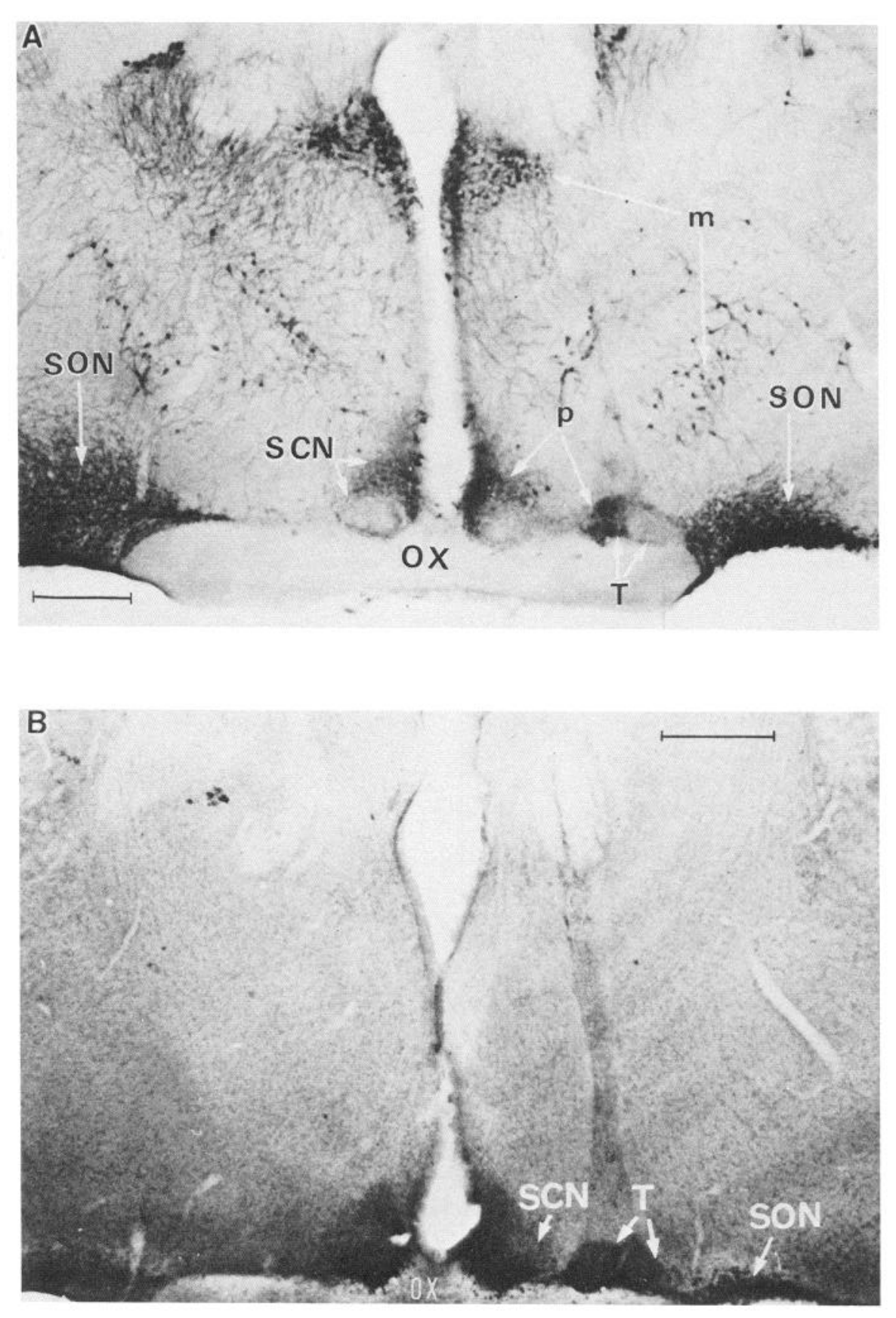

Thus, it was suggested that central catecholaminergic neurons play an important role in the initiation of hypertension in SHR. Impairment of other peptidergic mechanisms such as angiotensin (Casto and Phillips, 1985; Plunkett and Saavedra, 1985), GABA (Catelli and Sved, 1988), and serotonin (Koulu et al., 1986) seems also to be responsible for the development and/or maintenance of hypertension in this strain. Thus, there is a possibility that the graft influenced brainstem nuclei through HTH pathways (Swanson et al., 1986) to cause the long-lasting hypertension in the G-SHR.

In addition to the elevation in $\mathrm{BP}$, we found that transplantation of HTH tissue from genetically hypertensive (SHR) embryos to adult normotensive rats (WKY) resulted in a wide spectrum of abnormalities in the host animal that are similar

Figure 6. Sections through HTH taken 2 months after transplantation. $A$, Transplant $(T)$ from embryonic day 16 SHR located in the third ventricle $(V)$ of a WKY rat. Clusters of VP-immunopositive neurons are indicated by arrows. $O X$, optic chiasm. Scale bar, $100 \mu \mathrm{m}$. B, Transplant $(T)$ from embryonic day 16 WKY rat located in the third ventricle $(V)$ of WKY rat. A cluster of VP-immunopositive neurons is indicated by arrows. Scale bar, $120 \mu \mathrm{m}$. $C$ and $D$, High magnification of HTH transplant taken $300 \mu \mathrm{m}(C)$ and $400 \mu \mathrm{m}(D)$ rostrally from the brain section shown in $A$, stained for VIP and VP, respectively. Here, the graft is attached to the ventricle walls $(V W)$. Some immunopositive fibers appear to run into the host brain (arrows). Scale bars, $50 \mu \mathrm{m}$ for $C$ and $D$. 

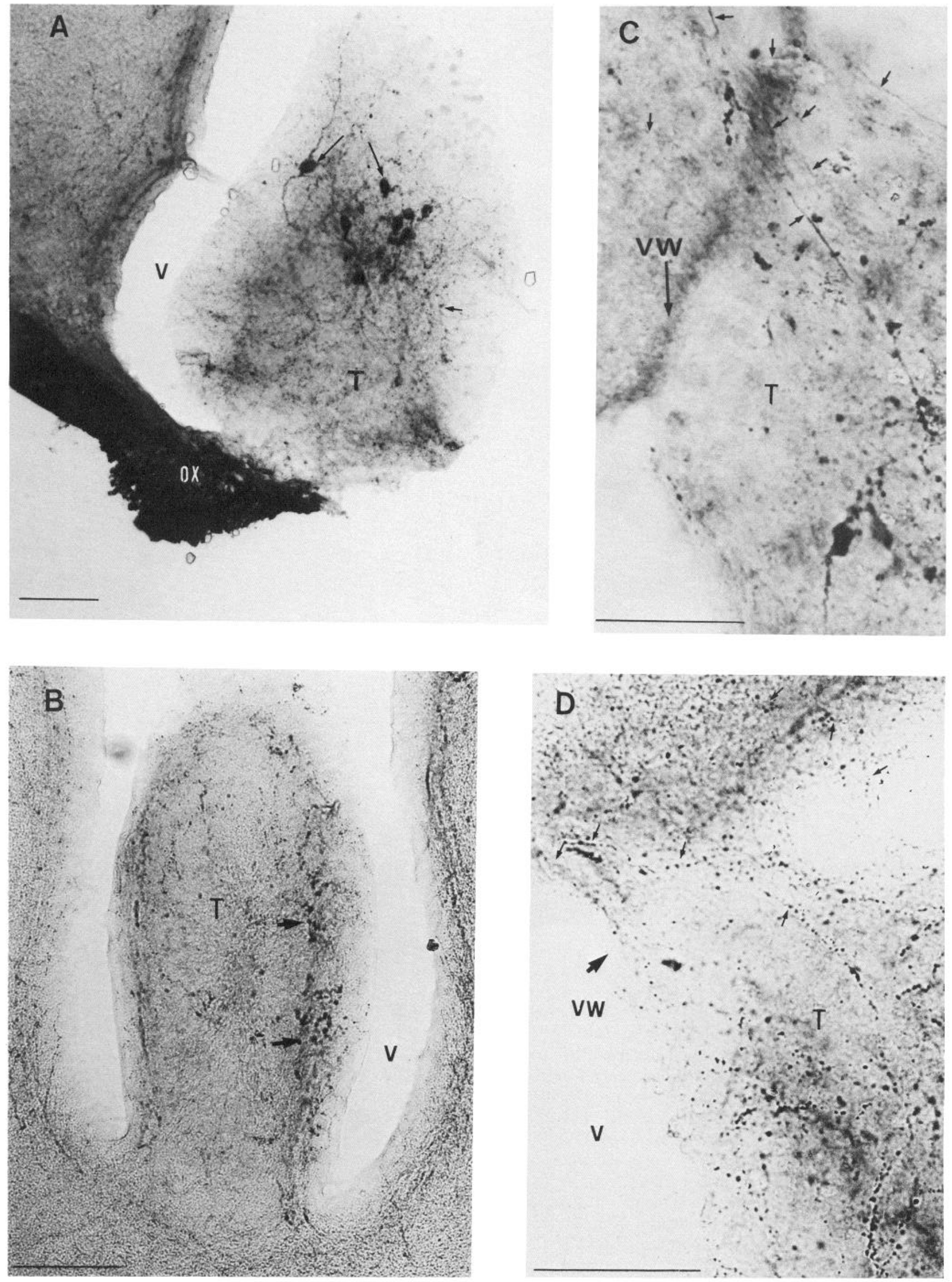
Figure 7. Coronal section through HTH stained for VP to show viable cells and fibers. The section was taken 1 month after transplantation. $A$, Highmagnification view of the transplant $(T)$ from embryonic day 16 SHR. Solid arrows indicate positive fibers. Open arrows indicate VP-positive neurons. Scale bar, $125 \mu \mathrm{m}$. $B$, Low-magnification view of the transplant shown in $A$ (corresponding to boxed area). The transplant was located in the third ventricle (V). The section was preincubated with oxytocin to insure VP specificity. Scale bar, $500 \mu \mathrm{m}$.

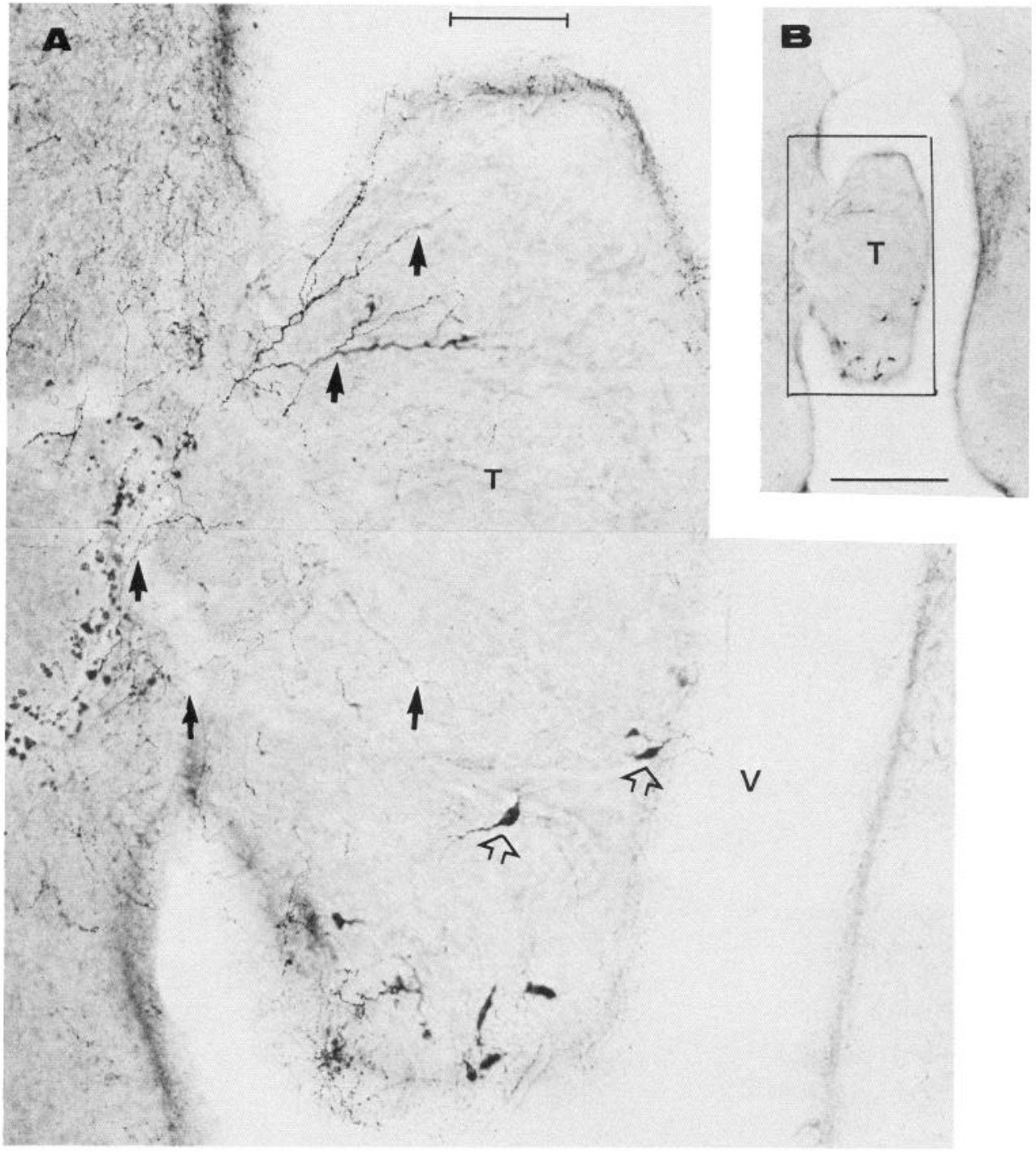

to those found in the native SHR (Crofton et al., 1978; Sladek et al., 1986; Grassi de Gende, 1988; Michel et al., 1988; Avidor et al., 1989). These findings emphasize the important role of the $\mathrm{HTH}$ in the regulation of BP.

After grafting, the cardiac hypertrophy was significantly more severe in the G-SHR hosts than in the untreated SHR group, despite the fact that the latter exhibited higher BP. Several studies (Sen et al., 1974, 1976; Cutilletta et al., 1978; Grassi de Gende, 1988) have reported poor correlations of left ventricle mass with BP levels in the SHR. This dissociation was based on both the development of hypertrophy before the increase in BP (Cutilletta et al., 1978; Sen and Tarazi, 1986) and the failure of antihypertensive drugs to reverse the increase of the left ventricular mass in the SHR strain (Sen et al., 1976). Two main factors were proposed for the modulation of left ventricular hypertrophy: (1) The levels of cardioadrenergic activity (Sen and Tarazi, 1983) and of peripheral angiotensin II (Michel et al., 1988), and (2) a local growth factor isolated from SHR hearts (Sen and Tarazi, 1986). It is possible that the rapid elevation of BP that was induced in the G-SHR rats accelerated the influence of these factors.
Pharmacological, electrophysiological, and behavioral studies indicate that systems controlling cardiovascular functions are closely coupled with systems modulating the perception of pain. Thus, induction of hypertension in rats is associated with a reduced response to noxious thermal (e.g., the hot-plate test) or mechanical stimuli (e.g., paw pressure; Zamir et al., 1980). We have found that the transplantation of HTH SHR tissue into WKY rats also resulted in a reduced response of the host to noxious stimuli. Although it has been shown that the brain of hypertensive animals contains increased levels of opioid peptides (Martucci and Hahn, 1979; Morris et al., 1981), the underlying mechanisms involved in this hypoalgesia have not been fully elucidated.

The SHR strain is characterized by many neuroendocrine changes referred to the HTH (Versteeg et al., 1976; Ciriello and Calaresu, 1980; Morris and Keller, 1982; Millett et al., 1986). In our study, we focused on changes in VIP gene expression. Although the role of this peptide in BP regulation is not known, we previously demonstrated increased VIP gene expression in the HTH of hypertensive animals (Avidor et al., 1989). Direct intracerebroventricular injections of VIP induce a dose-depen- 

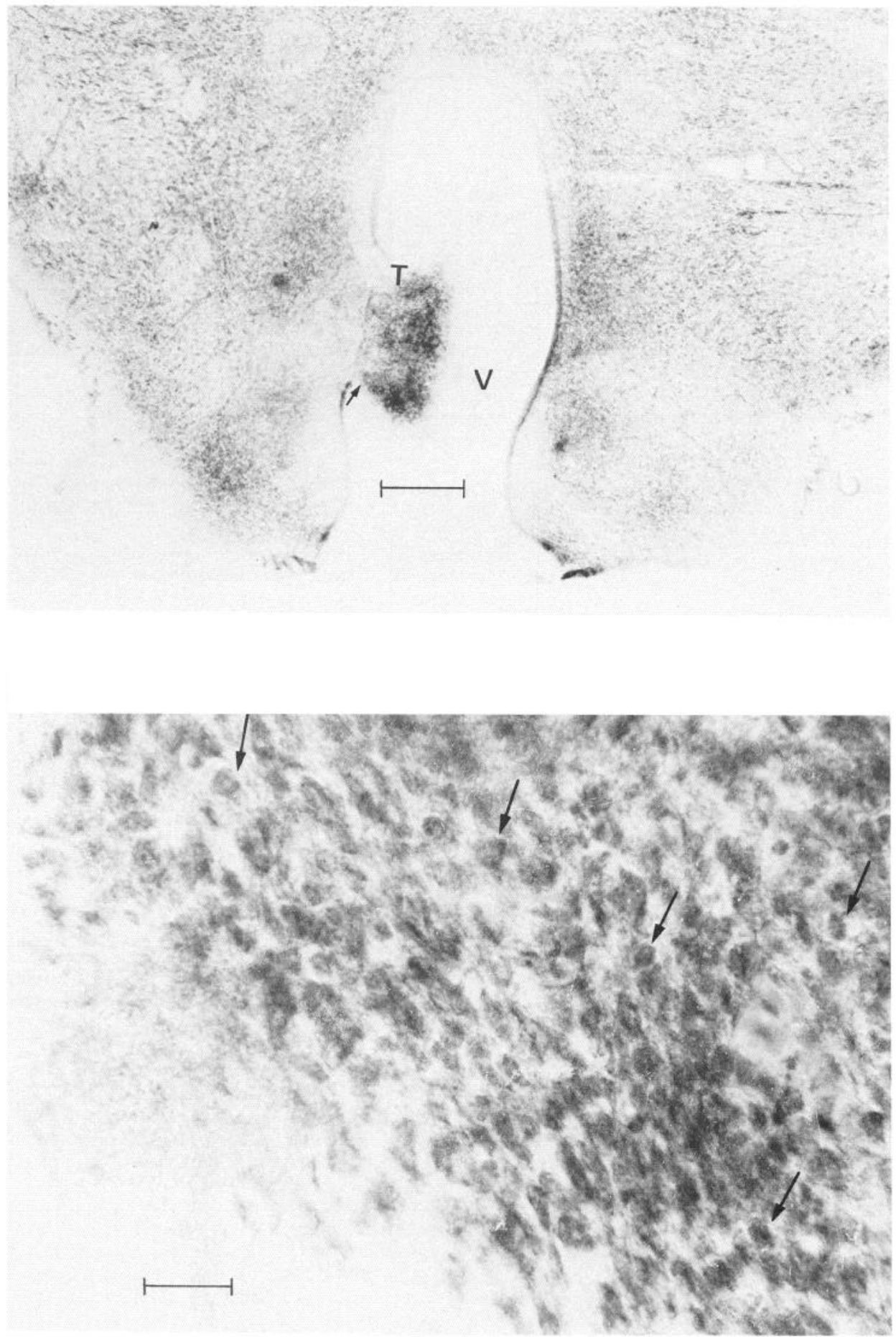

Figure 8. Bright-field photomicrograph of Nissl-stained coronal section neighboring ( $400 \mathrm{~mm}$ rostrally) section shown in Figure 7. Upper panel, Lowpower magnification showing the transplanted tissue $(T)$ in the third ventricle (V). Scale bar, $500 \mu \mathrm{m}$. Lower panel, High-magnification view of the area indicated by an arrow in A. Arrows in lower panel point to clustering of neuronal somata in the transplant. Scale bar, $25 \mu \mathrm{m}$. dent pressor response (Casto and Phillips, 1983). These findings indicate a possible involvement of VIP in the production of hypertension in the SHR. In the present study, we show that implantation of SHR HTH tissue in the WKY increases the VIP gene expression of the host brain. Further investigation is still required to elucidate whether this increase contributes to the production of high BP.

VIP is a potent vasodilator that exerts a direct effect on the cerebral arteries (Wei et al., 1980; Lee et al., 1984). Immunohistochemical staining for VIP in the rats with $\mathrm{HTH}$ grafts showed that the axonal processes could often be traced to the vicinity of blood vessels. This suggests that the grafted VIP cells either innervated the blood vessels or released neuromodulators onto them. The increased concentration of VIP mRNA could result in greater biosynthesis of the vasodilating polypeptide, which may be a general mechanism acting to protect blood vessels against the adverse effects of high BP.

A preliminary finding from our lab (R. Eilam, R. Malach, and $\mathrm{M}$. Segal, unpublished observations) indicates the existence of VIP-immunoreactive staining of the magnocellular perikarya 
in the supraoptic nucleus (SON) of grafted rats and SHRs. This staining was completely eliminated by pretreatment with synthetic VIP. Immunopositive staining of parvocellular perikarya for VIP has been described in the PVN of colchicine-treated Brattleboro rats. In contrast to these findings, immunopositive staining of parvocellular PVN neurons was found in the colchicine-treated Sprague-Dawley and Long-Evans rats only during lactation or after adrenalectomy (Mezey, 1986). Recently, the presence of VIP-immunopositive magnocellular perikarya has been demonstrated in both the PVN and the SON of the Mongolian gerbils (Mikkelsen and Moller, 1988). The elevation of VIP mRNA in the hypertensive rats, and the presence of VIP-containing neurons in the HTH, SON, and PVN in animal strains under certain physiological conditions, may indicate a role for VIP in circulatory control. Further studies are required to clarify this function.

It has been found that VP, present in the HTH and brain stem, interacts with baroreceptors, producing a further decrease in heart rate and cardiac output in response to $\mathrm{BP}$ elevation (Liard et al., 1981; Izdebska et al., 1982; Chiu and McNeill, 1985). On the basis of preliminary studies in our laboratory, we suggest that the number of VP-stained neurons and processes was drastically reduced in the immediate vicinity of the G-SHR grafts, in comparison with their number in the control graft (see Fig. 5A). A decrease in the HTH concentration of VP has been demonstrated in SHR (Crofton et al., 1978; Morris et al., 1981). Therefore, the gradual disappearance of VP from the graft area of the G-SHR may indicate that the graft is involved in this process, which could be related to the induction of hypertension.

The finding that the grafts in our experiments were of relatively small size may be attributed to the fact that the host HTH tissue was not destroyed prior to implantation, nor were cavities formed to promote growth. On the other hand, the small size of the grafts supports our assumption that the elevated BP of the hosts was not the result of mechanical pressure on the HTH region.

The present studies reveal that characteristic properties of hypertensive rats can be transferred by transplantation of the HTH into the brain of normotensive animals and thus support the assumption that the HTH may be the primary site involved in producing spontaneous hypertension in mammals. Further research on the interaction between the graft and its immediate surroundings may help to elucidate the processes involved in the development of hypertension.

\section{References}

Avidor R, Eilam R, Malach R, Gozes I (1989) VIP-mRNA is increased in hypertensive rats. Brain Res 503:304-307.

Bianchi G, Fox U, Di Francesco GF, Giovanetti AM, Pagetti D (1974) Blood pressure changes produced by kidney cross-transplantation between spontaneously hypertensive rats and normotensive rats. Clin Sci Mol Med 47:435-448.

Biegon A, Israeli M (1978) Quantitative autoradiographic analysis of the effect of electroconvulsive shock on serotonin-2 receptors in male and female rats. $J$ Neurochem 48:1386-1391.

Bloch B, Popovici T, Le Guellec D, Normand E, Chuaham S, Guitteny $\mathrm{AF}$, Bohlen $\mathrm{P}$ (1986) In situ hybridization hystochemistry for the analysis of gene expression in the endocrine and central nervous system tissues: a 3 year experience. J Neurosci Res 16:183-200.

Boer GJ, Gash DM, Dick L, Schluter N (1985) Vasopressin neuron survival in neonatal Brattleboro rats: critical factors in graft development and innervation of the host brain. Neuroscience 15:10871109.

Calaresu FR, Faiers AA, Morgenson GJ (1975) Central neural regu- lation of heart and blood vessels in mammals. Prog Neurobiol 5:333.

Casto R, Phillips MI (1983) The opposite effects of central and peripheral vasoactive intestinal polypeptide on blood pressure in rats. Neurosci Lett 38:169-173.

Casto R, Phillips MI (1985) Neuropeptides action in nucleus tractus solitarius: angiotension specificity and hypertensive rats. Am J Physiol 249:R341-R347.

Catelli JM, Sved AF (1988) Enhanced pressor response to GABA in the nucleus tractus solitarii of the spontaneously hypertensive rat. Eur J Pharmacol 151:243-248.

Chiu EK, McNeill JR (1985) Vasopressin withdrawal produces hypertension in spontaneously hypertensive rat. Am J Physiol 249:H193H197.

Ciriello J, Calaresu FR (1980) Role of paraventricular and supraoptic nuclei in central cardiovascular regulation in the cat. Am J Physiol 239:R137-R142.

Crofton JT, Share L, Shade RE, Allen C, Tarnowski D (1978) Vasopressin in the rat with spontaneous hypertension. Am J Physiol 235:H361-H366.

Cutilletta AF, Benjamin M, Culpepper WS, Oparil S (1978) Myocardial hypertrophy and ventricular performance in the absence of hypertension in the spontancously hypertensive rats. J Mol Cell Cardiol 10:689-693.

Eckenstein F, Baughman RW (1984) Two types of cholinergic innervation in cortex one co-localized with vasoactive intestinal polypeptide. Nature 309:153-155.

Faden AI, Feuerstein G (1983) Hypothalamic regulation of the cardiovascular and respiratory systems: role of specific opiate receptors. Br J Pharmacol 79:997-1002.

Fukushima M (1968) Histometric and histochemical studies of the hypothalamo-hypophyseal system of spontaneously hypertensive rats and rats with experimental hypertension. Jpn Circ J 33:485-516.

Gozes I, Avidor R, Biegon A, Baldino F Jr (1989) Lactation elevates vasoactive intestinal peptide messenger ribonucleic acid in the rat suprachiasmatic nucleus. Endocrinology 124:181-186.

Grassi de Gende AO (1988) Evaluation of right and left ventricular size in SHR-Wistar hybrids. Am J Physiol 255:H587-H591.

Guyton AC (1987) Renal function curve-a key to understanding the pathogenesis of hypertension. Hypertension 10:1-6.

Izdebska E, Jodkowski J, Trzebski A (1982) Central influence of vasopressin on baroreceptor reflex in normotensive rais and its lack in spontaneously hypertensive rats (SHR). Experimentia 38:594-595.

Koulu M, Saavedra JM, Niwa M, Scheinin M, Linnoila M (1986) Association between increased serotonin metabolism in rat brainstem nuclei and development of spontaneous hypertension. Brain Res 371: $177-181$

KrukoffTL, Weigel MA (1989) Metabolic alteration in discrete regions of the rat brain during development of spontaneous hypertension. Brain Res 499:1-6.

Lang RE, Rascher W, Unger T, Ganten D (1981) Reduced content of vasopressin in the brain of spontaneously hypertensive as compared to normotensive rats. Neurosci Lett 23:199-201.

Lee TJ, Saito A, Berezin I (1984) Vasoactive intestinal polypeptidelike substance: the potential transmitter for cerebral vasodilation. Science 224:898-901.

Liard JF, Deriaz O, Tschopp M, Schoun J (1981) Cardiovascular effects of vasopressin infused into the vertebral circulation of conscious dogs. Clin Sci 61:345-347.

Martucci CP, Hahn EF (1979) Brain opiate receptor concentrations are increased in adult spontaneously hypertensive rats. Endocrinol Res Commun 6:291-297.

Mezey E (1986) Vasoactive intestinal polypeptide immunopositive neurons in the paraventricular nucleus of homozygous Brattleboro rats. Neuroendocrinology 42:88-90.

Michel JB, Salzmann JL, Cerol ML, Dussaule J-C, Azizi M, Corman B, Camilleri J-P, Corvol P (1988) Myocardial effect of converting enzyme inhibition in hypertensive rats. Am J Med 84:12-21.

Mikkelsen JD, Moller M (1988) Vasoactive intestinal peptide in the hypothalamohypophysial system of the Mongolian gerbil. J Comp Neurol 273:87-98.

Millett JA, Holland SM, Alaghband-Zadeh J, de Wardener HE (1986) Na-K-ATPase-inhibiting and glucose-6-phosphate dehydrogenasestimulating activity of plasma and hypothalamus of Okamoto spontaneously hypertensive rats. J Endocrinol 108:69-73. 
Morris M, Keller M (1982) A specific deficiency in paraventricular vasopressin and oxytocin in the spontaneously hypertensive rat. Brain Res 249:173-176.

Morris M, Wren JA, Sundberg DK (1981) Central neural peptides and catecholamines in spontaneous and DOCA/salt hypertension. Peptides 2:207-211.

Nagaoka A, Lovenberg W (1977) Regional changes in the activities of aminergic biosynthetic enzymes in the brain of hypertensive rats. Eur J Pharmacol 43:297-306.

Nelson DO, Boulant JA (1981) Altered CNS neuroanatomical organization of spontaneously hypertensive (SHR) rats. Brain Res 226: 119-130.

Plunkett LM, Saavedra JM (1985) Increased angiotension II binding affinity in the nucleus tractus solitarius of spontaneously hypertensive rats. Proc Natl Acad Sci USA 82:7721-7724.

Randich A, Maixner W (1984) Interactions between cardiovascular and pain regulatory systems. Neurosci Biobehav Rev 8:343-367.

Saavedra JM, Grobecker H, Axelrod J (1978) Changes in central catecholaminergic neurons in the spontaneously (genetic) hypertensive rat. Circ Res 42:529-534.

Segal M, Greenberger V, Pearl E (1989) Septal transplants ameliorate spatial deficits and restore cholinergic functions in rats with a damaged septo-hippocampal connection. Brain Res 500:139-148.

Sen S, Tarazi RC (1983) Regression of myocardial hypertrophy and influence of adrenergic system. Am J Physiol 244:H97-H101.

Sen S, Tarazi RC (1986) Cardiovascular hypertrophy in spontaneously hypertensive rats. J Hypertens 4:S123-S126.

Sen S, Tarazi RC, Bumpus FM (1974) Cardiac hypertrophy in the spontaneously hypertensive rats. Circ Res 35:775-781.
Sen S, Tarazi RC, Bumpus FM (1976) Biochemical changes associated with development and reversal of cardiac hypertrophy in spontaneously hypertensive rats. Cardiovasc Res 10:254-261.

Sladek CD, Blair ML, Chen YH, Rockhold RW (1986) Vasopressin and renin response to plasma volume loss in spontaneously hypertensive rats. Am J Physiol 250:H443-H452.

Swanson LW, Sawchenko PE (1980) Paraventricular nucleus: a site for the integration of neuroendocrine and the autonomic mechanisms. Neuroendocrinology 31:410-417.

Swanson LW, Sawchenko PE, Lind RW (1986) Regulation of multiple peptides in CRF parvocellular neurosecretory neurons: implication for the stress response. Prog Brain Res 68:169-188.

Versteeg DHG, Palkovits M, Van der Gugten J, Wijnen HLJM, Smeet GWM, de Jong W (1976) Catecholamine content of individual brain regions of spontaneously hypertensive rats (SH-rats). Brain Res 112: $429-434$.

Wei EP, Kontos HA, Said SI (1980) Mechanism of action of vasoactive intestinal polypeptide on cerebral arterioles. Am J Physiol 239: H765-H768.

Wijnen HJ, Palkovits M, de Jong W, Versteeg DHG (1978) Elevated adrenaline content in nuclei of medulla oblongata and the hypothalamus during the development of spontaneous hypertension. Brain Res 157:191-195.

Zamir N, Simantov R, Segal M (1980) Pain sensitivity and opioid activity in genetically and experimentally hypertensive rats. Brain Res 184:299-310. 